Methods ResNet50 pre-trained on ImageNet was selected as our CNN main framework. We collected 5,875 endoscopic images and 16 full-length videos from patients with UC who underwent colonoscopy from January 2017 through March 2021as the dataset. The scoring system was trained by these images and could predict the Mayo score and UCEIS score by the feature it has learned on the training set. In addition, by using the predicted outcome, the CAD could show a ribbon in the shape of the colon which can reflect the conditions of the inflammation (location, severity). The scoring system is composed of clarity cleaning, similarity judgement and score prediction networks. This corresponding relation which combines the exiting endoscopy scores prediction, helps the system to output a new score with weighted calculation, as well as the lesions' location and severity ratio.

Results Two clinical reviewed our consistency statistics. The primary evaluation metric we used is quadratic weighted kappa (QWK). The trained CNN got high levels with 90.2\% accuracy in the Mayo-scored task, while the QWK coefficient was 0.906 (95\%CI, 0.880-0.933). The metrics of UCEIS-scored task were inspiring of which accuracy were 90.7\%, 84.6\%, 77.7\%, QWK coefficient were 0.887 (95\%CI, 0.860-0.915), 0.876 (95\%CI, 0.844-0.907), 0.816 (95\%CI, 0.739-0.892) for vascular pattern, erosions-andulcers and bleeding. Meanwhile, the CNN identified 16 full-length endoscopy videos and evaluated 1,360 local information from 100 intestine segments. The results of these localized intestinal tissue marked by the scored system were distributed in four ranges, and the quantities were 772(0.0-0.4), 124(0.72-2.35), 206(3.2-4.0), 258(5.28.4). It performed desirable compared with the clinical diagnosis.

Conclusions The results of objective evaluations of UC based on this scoring system appeared acceptable. Compared with the previous scored methods (only refer to the most severe lesion and ignored other mild parts), this work is of clinical significance and will help clinicians by supplying a good capacity in identifying endoscopy data and stimulating the colon's inflammatory environment.

\section{IDDF2021-ABS-0208 DEVELOPMENT OF AN AUTOMATIC SYSTEM TO FAST QUANTIFY MARKING AND INCISION DURING ENDOSCOPIC SUBMUCOSAL DISSECTION}

\footnotetext{
${ }^{1}$ Shuntian Cai ${ }^{*},{ }^{2}$ Jiacheng Wang, ${ }^{2}$ Minjie Wang, ${ }^{3}$ Xiaoning Yang, ${ }^{2}$ Lingfeng Zhong, ${ }^{2}$ Jianlin Ren, ${ }^{2}$ Liansheng Wang, 'Hongzhi Xu. 'Department of Gastroenterology, Zhongshan Hospital Affiliated to Xiamen University, China; ${ }^{2}$ Department of Computer Science, Xiamen University, China; ${ }^{3}$ Department of Digestive Diseases, School of Medicine, Xiamen University, China
}

\subsection{6/gutjpl-2021-IDDF.175}

Background Facilitating accurate location of marking points and circumferential incision in the mucosa at a certain distance outside the boundary of the lesion is important to ensure the quality of endoscopic submucosal dissection (ESD). However, the decision-making of different endoscopists has a large variance due to subjective experience, leading to difficulty in quality control of ESD. To tackle this problem, this study aims at utilizing an intelligent automatic system to give real-time guidance during the operation.

Methods We built an Artificial Intelligence (AI) assistant system that can detect the lesion boundary and marked points in real-time to quantify the location of marking points and cutting path during ESD of early esophageal cancer(EEC). This system was composed of two major components, that were the marking points suggestion module and the cutting line suggestion module. We collected 499 endoscopic images with Lugol's staining of EEC from 20 cases and 3397 detailed images of ESD from 12 cases. With these images, we built datasets for the evaluation of lesion boundary detection and marked points detection. Then, the algorithm was built to display the marking position and the mucosal incision position.

Results We conducted the experiments, including two parts. The first one was to assess the real-time boundary detection performance and marked points detection performance. Specifically, we achieved a satisfactory real-time lesion segmentation performance with Dice score of $96.1 \%$, and a good marked points detection performance with average precision (AP) 50 of $72.1 \%$. On the basis of boundary detection, our algorithm can automatically display the marking position at a certain distance (such as $0.3 \mathrm{~cm}$, which can be adjusted as needed) outside the boundary of the lesion. After the guidance of marking points, the automatic system can detect the marked points and display the mucosal cutting line at a certain distance outside the marked points.

Conclusions Suggesting position of marking and incision were successfully achieved during ESD with our automatic system, which can improve consistency of standard ESD procedure among different level hospitals.

\section{IDDF2021-ABS-0211 CO-OCCURRENCE OF GUT MICROBIOTA DYSBIOSIS AND BILE ACID METABOLISM ALTERATION IS ASSOCIATED WITH PSYCHOLOGICAL DISORDERS IN CROHN'S DISEASE}

${ }^{1}$ Xuefeng Gao*, ${ }^{1}$ Lijuan Feng, ${ }^{2}$ Nan Zhou, ${ }^{2}$ Zichun Li, ${ }^{2}$ Dongni Fu, ${ }^{3}$ Ying Guo, ${ }^{2}$ Xiaowei Liu. ${ }^{1}$ Department of Gastroenterology and Hepatology, Shenzhen University General Hospital, China; ${ }^{2}$ Department of Gastroenterology, Xiangya Hospital, Central South University, China; ${ }^{3}$ Department of Clinical Pharmacology, Xiangya Hospital, Central South University, China

\subsection{6/gutjnl-2021-IDDF.176}

Background Metabolites of the gut microbiota interviewing with signals derived from the host immune, endocrine, and neuronal systems intertwining may influence the development of mucosal injury and inflammation and impacts also distal central nervous system, underlying the psychophysiological vulnerability of Crohn's disease (CD) patients. This study aims to elucidate the relationships between bile acid metabolism, gut microbiota, and psychological comorbidity in $\mathrm{CD}$.

Methods A total $39 \mathrm{CD}$ patients and 14 healthy controls were enrolled in this study. The psychological status of the participants was accessed by using Zung Self-rating Depression Scale (SDS) and Self-rating Anxiety Scale (SAS). Bile acids in the feces and serum samples were quantified by using liquid chromatography-tandem mass spectrometry (LC-MS/MS). 16S 